\title{
Microarray Analysis of Transcriptomic Response of Escherichia coli to Nonthermal Plasma-Treated PBS Solution
}

\author{
Suresh G. Joshi' ${ }^{1,2}$, Adam Yost ${ }^{2}$, Siddharth S. Joshi², Sankar Addya ${ }^{3}$, Garth Ehrlich1, \\ Ari Brooks ${ }^{1,4}$ \\ ${ }^{1}$ Center for Surgical Infection and Biofilm, College of Medicine, Drexel University, Philadelphia, USA \\ ${ }^{2}$ School of Biomedical Engineering, Science and Health, Drexel University, Philadelphia, USA \\ ${ }^{3}$ Kimmel Cancer Center, Thomas Jefferson University, Philadelphia, USA \\ ${ }^{4}$ Department of Surgery, University of Pennsylvania Health System, Philadelphia, USA \\ Email: Suresh.Joshi@drexelmed.edu, surejoshi@yahoo.com
}

Received 13 January 2015; accepted 27 January 2015; published 30 January 2015

Copyright (C) 2015 by authors and Scientific Research Publishing Inc.

This work is licensed under the Creative Commons Attribution International License (CC BY).

http://creativecommons.org/licenses/by/4.0/

(c) (i) Open Access

\begin{abstract}
We developed a technique of generating nonthermal atmospheric plasma-activated solution that had broad-spectrum antibacterial properties. Plasma-activated phosphate-buffered saline (PBS) causes rapid inactivation of bacteria following generation of oxidative stress. However, dose optimization requires understanding of cellular mechanisms. The objective of this study was to explore genome-wise response to develop gene expression profile of Escherichia coli using DNA microarray following exposure to plasma-activated PBS solution. Upon exposure to plasma-treated PBS solution, $E$. coli cells had differentially expressed genes involved in oxidative stress, and cell envelope and membrane associated porin and transporters. The genes involved in house-keeping and metabolism, energy generation, motility and virulence were conversely downregulated. This is the first report which demonstrates a severe oxidative stress induced in $E$. coli cells in response to an exposure to nonequilibrium nonthermal dielectric-barrier discharge plasma-activated PBS solution, and the genes that are responsive to reactive oxygen species appeared to play a role in cellular stress. Such studies are important to identify targets of inactivation, and to understand plasmatreated solution and bacterial cell interactions.
\end{abstract}

\section{Keywords}

Antibacterial Solution, Disinfection, Escherichia coli, Gene Expression, Indirect Plasma, Microarray, Nonthermal Plasma, Transcriptomics

\footnotetext{
${ }^{*}$ Corresponding author.
}

How to cite this paper: Joshi, S.G., Yost, A., Joshi, S.S., Addya, S., Ehrlich, G. and Brooks, A. (2015) Microarray Analysis of Transcriptomic Response of Escherichia coli to Nonthermal Plasma-Treated PBS Solution. Advances in Bioscience and Biotechnology, 6, 49-62. http://dx.doi.org/10.4236/abb.2015.62006 


\section{Introduction}

Nonthermal nonequilibrium atmospheric plasma (plasma) is being investigated for disinfection and sterilization processes in biology and medicine. Since last decade, several publications appeared which stressed the advantages of this technique over traditional disinfection techniques [1] [2]. The direct plasma application technique is a little challenging to delicate surfaces and tissues, which are likely to be damaged by high energy electrons and UV photons generated during direct bombardment of plasma [3]-[6]. In addition, the distance between plasma generating probe and the surface being treated is critical, and determines the dose and efficacy of plasma.

Recently, we have demonstrated a technique of applying plasma indirectly wherein the plasma generating probe doesn't come in contact with such surface or the surface of skin or mucous membranes, and is highly portable and does not require gas or air cylinders and the associated assembly (unlike jet or afterglow plasma technique). We have developed plasma-treated solutions that retain strong antimicrobial property for up to two years of time period [7]. The antibiotic-like solutions are fast acting and require only about 180 seconds of plasma treatment for activation of antibacterial property, and less than 15 min of contact (holding) time is sufficient to inactivate bacterial pathogens [7]. A plasma-activated phosphate-buffered saline (PBS) solution is one of the strong oxidative biocidal agents we generated, which has powerful antibacterial and antibiofilm properties. Upon plasma exposure, certain oxidizing chemical species are produced in PBS solutions which are responsible for rapid inactivation of bacteria [7] [8]. Based on our preliminary observations, we anticipate that oxidative stress is responsible for rapid cell death, but the exact mechanism of bacterial inactivation is not known. We also anticipate that plasma-activated PBS solution may have multiple targets, primarily related to oxidative stress-mediated changes. A systematic genome-wise analysis is required to define such targets of inactivation.

Genomic analysis is one of the most favored approaches of study underlying genetic mechanisms of inactivation and regulatory response of genes that govern cell death. Cellular defenses have their thresholds and beyond this limit cells cannot survive stress conditions. Escherichia coli is one of the suitable model bacteria for this study whose whole genome sequence is available and metabolic pathways are well studied [3] [9]. DNA microarray is a widely used molecular technique which allows differentiating gene expression under various conditions, to monitor patterns of global gene expression, and thus helps in understanding cellular processes. In addition, microarrays are much more convenient to work with than DNA/RNA membrane blotting [10]. Aim of this study was to explore the gene expression profile responses of global genes in $E$. coli that occur upon exposure to plasma-activated antimicrobial PBS solution, to predict related transcriptomic changes.

\section{Materials and Methods}

\subsection{Bacterial Culture and Condition}

Escherichia coli (ATCC-29522) was used for this study. The culture was developed by inoculation of a single isolated colony from overnight grown trypticase soy agar plate into $10 \mathrm{ml}$ of trypticase soy broth (TSB) and incubated at $37^{\circ} \mathrm{C}$ in a stationary incubator. On the following day, the culture was reinoculated and the growth monitored by taking optical density $\left(\mathrm{OD}_{600}\right)$ of the culture aliquots.

\subsection{Chemical Solution, Plasma Setting, and Treatment Conditions}

Phosphate-buffered saline solution (PBS; Sigma; $150 \mathrm{mmol} / \mathrm{L}$ sodium chloride and $150 \mathrm{mmol} / \mathrm{L}$ sodium phos-

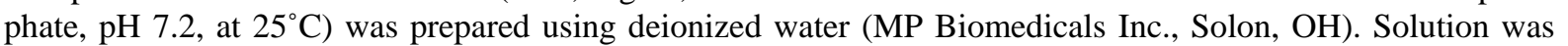
freshly prepared, $0.22 \mu$ filter sterilized, aseptically handled; and aliquot either used fresh or stored at $-20^{\circ} \mathrm{C}$ for subsequent experiments. Similarly, $\alpha$-tocopherol (vitamin E; $200 \mathrm{mM}$ ), thiourea and catalase (all from SigmaAldrich, St. Louis, MO, USA) were prepared as stock solutions in untreated PBS buffer, filter sterilized, and used at predetermined concentrations ( $\alpha$-tocopherol, $200 \mathrm{mM}$; thiourea, $50 \mathrm{mM}$; catalase, 200 units). Nonthermal plasma generator was used in this study, and the in-house built electrode and fluid chamber system was reported earlier by our laboratory [7]. The parameters were set to $11 \mathrm{kV}, 0.26 \mathrm{~W} / \mathrm{cm}^{2}, 1000 \mathrm{~Hz}$ pulse frequency, 2 $\mathrm{mm}$ of gap between the surface of fluid and DBD electrode. One milliliter of chemical solution was treated for 15 seconds through 180 seconds or left untreated [zero (0) second)], and used freshly.

\subsection{Bacterial Inactivation Assays}

Halfmilliliter $(0.5 \mathrm{ml})$ of PBS solution treated with plasma for various duration of treatment was mixed with 0.5 
$\mathrm{ml}$ of $E$. coli cell suspension ( $1 \mathrm{ml}$ culture of $0.2 \mathrm{OD}_{600}$ was centrifuged at 4000 revolutions per minute (RPM), cell pellet was washed twice with sterile PBS, and resuspended in $0.5 \mathrm{ml}$ of PBS), and held for $15 \mathrm{~min}$ of contact time. The cells were harvested by centrifugation, and resuspended in untreated PBS to proceed for XTT assay using XTT reagents (Molecular Probes) as described earlier [11] [12] to evaluate the treatment-time-dependent oxidative stress mediated antimicrobial efficacy of plasma-treated PBS and effect of ROS scavengers. A hydrogen peroxide $(0.3 \%)$ reagent and plasma untreated PBS solution were used as positive and negative controls respectively. The assays were repeated three times to confirm findings.

\subsection{Hydrogen Peroxide Detection Assay}

The amount of hydrogen peroxide retained in each sample was measured using Hydrogen Peroxide Detection Kit (National Diagnostics, Atlanta, GA) following manufacturer's protocol. In brief, a working solution of $20 \mathrm{ml}$ was prepared by combining two reagents supplied in the Kit, and the serial dilutions of standard hydrogen peroxide were tested to generate standard curve. In parallel, the undiluted or serially diluted plasma-activated PBS was prepared, and $\mathrm{H}_{2} \mathrm{O}_{2}$ detection assay performed in 96 wells plate in triplicate. The assay was repeated twice in triplicate and $\mathrm{H}_{2} \mathrm{O}_{2}$ concentrations determined as per manufacturer's protocol.

\subsection{Microarray Assays}

A predetermined (60 seconds) dose of plasma treatment was used to activate PBS solution. One ml exponential E. coli culture $\left(0.2\right.$ of $\mathrm{OD}_{600}$ ) was centrifuged at $4000 \mathrm{RPM}$ to harvest cells and the cell pellet was washed twice with sterile PBS and resuspended in $500 \mu \mathrm{l}$ of PBS. An equal amount (500 $\mu \mathrm{l}$ ) of plasma-treated or untreated PBS solution or $\mathrm{H}_{2} \mathrm{O}_{2}$ solution was then added to this cell suspension and the reaction mix was held for 15 min (contact time), after which cells were suspended in RNA later reagent (Qiagen, Valencia, CA) to stabilize RNA, and then were pelleted again by centrifugation, and subjected to cell lysis using RNeasy mini kits (Qiagen) following the manufacturer's protocol. RNA was isolated from E. coli cells exposed to either plasma-treated or untreated fluid or $\mathrm{H}_{2} \mathrm{O}_{2}$ solution, and quantified on a NanoDrop spectrophotometer (Thermo Scientific), followed by RNA quality assessment on an Agilent 2100 bioanalyzer (Agilent, Palo Alto, CA, USA). Amplification and labeling was performed using the Ovation Pico WTA-system V2 RNA amplification system (NuGen Technologies, Inc.). Briefly, 50 ng of total RNA was reverse transcribed using a chimeric cDNA/mRNA primer, and a second complementary cDNA strand was synthesized. Purified cDNA was then amplified with ribo-SPIA enzyme and SPIA DNA/RNA primers (NuGen). Amplified c-DNA was purified with Qiagen MinElute reaction cleanup kit. The concentration of Purified cDNA was measured using the NanoDrop. The cDNAs (2.5 $\mu \mathrm{g})$ were fragmented and chemically labeled with biotin to generate biotinylated cDNA using FL-Ovation cDNA biotin module V2 (NuGen). Affymetrix Genechip ${ }^{\circledR}$ E. coli Genome 2.0 array system (Affymetrix, Santa Clara, CA) was used. The product was hybridized with fragmented and biotin-labeled target $(2.5 \mu \mathrm{g})$ in $110 \mu \mathrm{l}$ of hybridization cocktail. Target denaturation was performed at $99^{\circ} \mathrm{C}$ for $2 \mathrm{~min}$ and then $45^{\circ} \mathrm{C}$ for 5 min, followed by hybridization for $18 \mathrm{~h}$. Arrays were then washed and stained using Gene chip Fluidic Station 450, and hybridization signals were amplified using antibody amplification with goat IgG and anti-streptavidin biotinylated antibody. A hydrogen peroxide $(0.3 \%)$ reagent and plasma untreated PBS solution were used as positive and negative controls respectively.

\subsection{Data Analysis}

Gene-Chips were scanned on an Affymetrix Gene Chip Scanner 3000, using Command Console Software. Background correction and normalization were done using Iterative Plier 16 with Gene Spring V11.5 software (Agilent). A list of differentially expressed genes (in fold) was generated for the genes whose transcription is significantly influenced, and the list was loaded into Ingenuity Pathway Analysis (IPA) 5.0 software

(http://www.ingenuity.com) to perform biological network and functional analyses. The transcript expression values of treated sample array (plasma-treated PBS) versus plasma untreated sample array were considered significant when the difference ratio was 1.2, and subsequently, we selected genes which were differentially expressed by $>2$ fold (against untreated samples). Experiments were repeated in triplicate, and a mean of fold expressions shown. 


\section{Results}

\subsection{Plasma-Activated PBS Doses, Bacterial Inactivation and Oxidative Stress}

The plasma generating setup used in these studies is published earlier [3] [7] [11]. Earlier, we reported that inactivation of bacteria is plasma-treatment (plasma energy)-dependent [7], and therefore only the representative growth inhibition curves, and the effect of ROS scavengers in restoring cells from oxidative stress are provided here (Figure 1). During optimization, we determined that $1 \mathrm{ml}$ of plasma-treated PBS solution for 60 seconds inactivate number of $E$. coli only by one quarter; hence we used predetermined 60 seconds treatment of PBS solution as sublethal dose for this microarray analysis study (details of the killing curves and colony count assay are published earlier and hence are not provided here). Figure 1 shows the representative graphs of plasmatreatment times-dependent $E$ coli growth inhibition, and the effect of anti-oxidants, $\alpha$-tocopherol (vitamin E), catalase, and thiourea on scavenging ROS, leading to significant protection of cells from cellular inactivation $\left({ }^{*} P \leq 0.05\right)$ as compared to corresponding non-scavenged conditions of both, 1 minute and 2 minute plasma-treated PBS. The hydrogen peroxide assay revealed that the amount of $\mathrm{H}_{2} \mathrm{O}_{2}$ generated in solution at 60 seconds (sub-lethal dose) was $0.42 \mathrm{mM}( \pm 0.03 \mathrm{mM})$ (an average of three readings).

\subsection{Plasma-Activated PBS Solution and Expression Profile of Global Genes}

By whole genome approach, E. coli genes were analyzed for their expression profile against the treatment response of plasma-activated PBS solution. The plasma-activated PBS solution chemistry is not yet fully understood. Based on our preliminary observations of generation of reactive oxygen species [3] [7] and the antioxidant mediated protection to E. coli cells (Figure 1), we thought of comparing with $\mathrm{H}_{2} \mathrm{O}_{2}$ treatment control. Looking at the large number of genes, only a change in expression of two-folds or more was considered for analysis. To minimize the number of false positives and enrich the transcriptomic response of differentially expressed genes, the signal intensities of $>50$ were considered (Figure 2(A)). The signals generated by plasmaactivated PBS and $\mathrm{H}_{2} \mathrm{O}_{2}$ were normalized against untreated cells in PBS (as the cells were suspended in untreated PBS for 15 min; and likely to exhibit some starvation stress). On exposure to plasma-activated PBS and $\mathrm{H}_{2} \mathrm{O}_{2}$, respectively 412 and 1272 genes differentially expressed, of which 218 were common among them when analyzed using pairwise comparison against untreated PBS condition (Figure 2(B)). Figure 3 is a heat map of the genes that are differentially expressed, and commonly involved in both, the plasma-activated PBS exposure and hydrogen peroxide exposure of cells.

Out of 412 genes differentially expressed upon plasma-activated PBS 230 were functionally defined genes, and rest 181 genes were either pseudo genes or hypothetical genes whose functional annotation is not defined. Total 120 genes were upregulated and 111 genes were downregulated. The findings of microarray assay of top

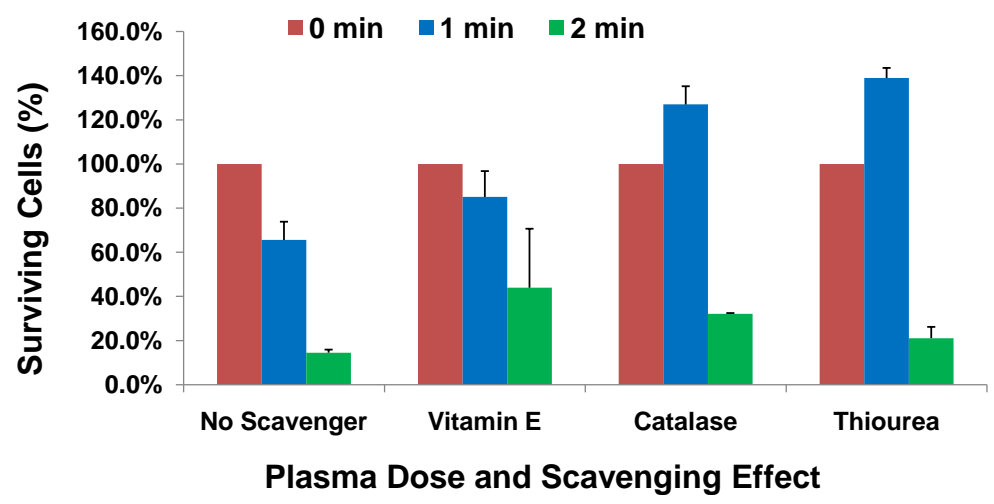

Figure 1. The kinetics of $E$. coli cell inactivation and the oxidative stress generated by plasma-treated PBS. A graphical presentation of XTT assay showing survival responses of $E$. coli against different plasma treatment time (at contact/holding time of $15 \mathrm{~min}$ ), and the scavengers of reactive oxygen species (ROS) giving significant protection from plasma-activated PBS mediated inactivation. " $P \leq 0.05$ against corresponding "no scavenger" conditions; $\mathrm{n}=3$. 


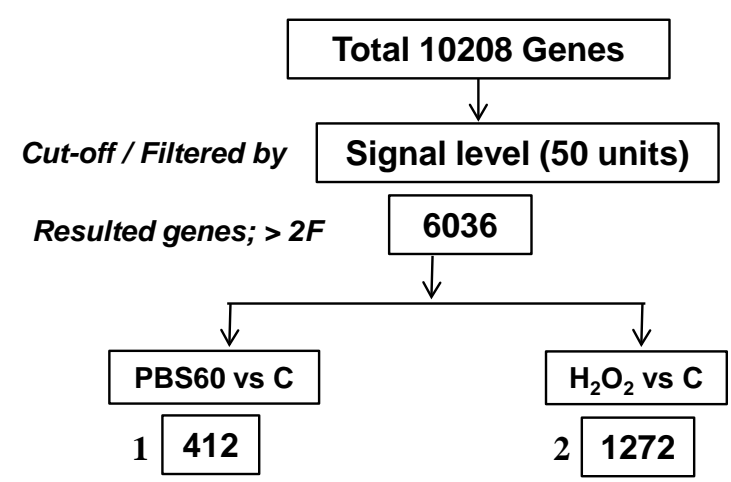

(a)

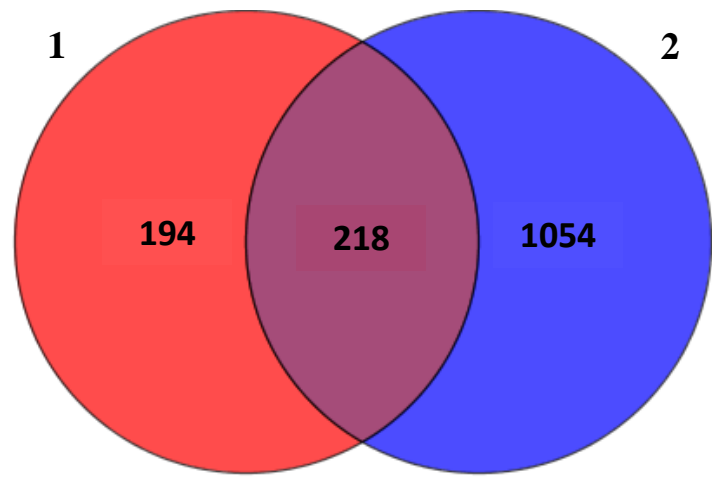

(b)

Figure 2. A schema of differentially expressed genes filtration that has $>2$ fold change (a) was adopted to reflect an enhancement of gene expression. A cut-off filtration of microarray fluorescing signals (50 units) was used to avoid false-positivity, which concurrently enhanced the gene expression profile; (b) A schematic presentation of differentially expressed genes of $E$. coli when cells exposed to plasma-activated PBS (PBS60) versus untreated PBS (circle 1; red), and cells exposed $\mathrm{H}_{2} \mathrm{O}_{2}$ versus untreated PBS (circle 2; blue). The 218 genes were differentially expressed, common in cells treated with $\mathrm{H}_{2} \mathrm{O}_{2}$ solution and plasma-activated PBS solution (PBS60), and were responsive to oxidative stress.

125 genes with their functional annotations which exhibited differential expression are grouped together and showed in Table 1. The transcriptomic response is categorized upon their cellular function, such as oxidative and other stress-related damage response, SOS response, nitrosative stress, cell envelop-related response, cell cycle/regulation, metabolism, transporter and Fe-S cluster assembly/cysteine synthesis. Differentially expressed genes represented about $4 \%$ of all genes of $E$. coli on chip. The fold increase is relative to untreated control. The upregulated genes were mostly related to stress response, iron-sulfur (Fe-S) cluster formation, cysteine biosynthesis pathway, biofilm formation, whereas downregulated genes were predominantly associated in general with cellular metabolism, ribosomal proteins, membrane proteins and porins, flagellum biosynthesis and motility.

\subsection{SOS Response and Universal Distress Signals}

SOS response is a global response to DNA damage wherein cell cycle is arrested and DNA repair (and/or mutagenesis) is induced, and includes the proteins related to Rec family (RecA, RecB, RecD, RecN). The SOS regulon involves protein RecA, responsible for inactivation of LexA repressor (and thus negatively regulated by LexA repressor protein dimmers), and is a complex of several genes that are coordinately expressed and involved in DNA repair. The RecA protein is under control of recombination regulator RecX; and RecN is required in DNA recombination. In present study, overall SOS genes were moderately upregulated ( $<2$ folds; recA, lexA, sulA, umuC, umuD). Only the genes, $r e c N$, $n f o, n r d E$ and $n r d F$ were upregulated by $>2$ folds (Table 1 ).

\subsection{Oxidative Stress Induced Genes}

We observed highest expression of oxyS transcript (28.8 fold upregulation), an indicator of a generation of severe oxidative stress. Hydrogen peroxide detoxifying genes such as kat $G$ (catalase), ahpC (peroxiredoxin), ahpF (hydroperoxidase) and $y g g P$ (putative oxidoreductase) were differentially expressed; respectively 19.3, 3.9, 7.0, and 3.1 folds (Table 1). All these genes are responsive to $\mathrm{H}_{2} \mathrm{O}_{2}$, suggests that hydrogen peroxide may be generated in E. coli cells. A transcriptional regulator of oxidative stress, SoxS was also upregulated (2.9 folds). SoxS is responsive to superoxide and singlet oxygen species. Out of three superoxide dismutases (SodA, MnSOD; SodB, FeSOD; and SodC, Cu/ZnSOD) that catalyze superoxide-like species, only transcript of sodA was upregulated. Other genes such as grxA (glutaredoxin-1) and $\operatorname{trx} C$ (thioredoxin-2) were also upregulated.

\subsection{Other Stress-Related Response and Metabolism and Regulatory Genes}

Reactive nitrogen species [13] —responsive genes such as hda, atpA, gapA, tufA, and napA were downregulated, whereas the genes of most of the Fe-S cluster assembly and cysteine synthesis pathway were significantly upregulated (Table 1). Genes, cadC and aceF, were upregulated by $<2$ folds, indicating that RNA mediated stress 


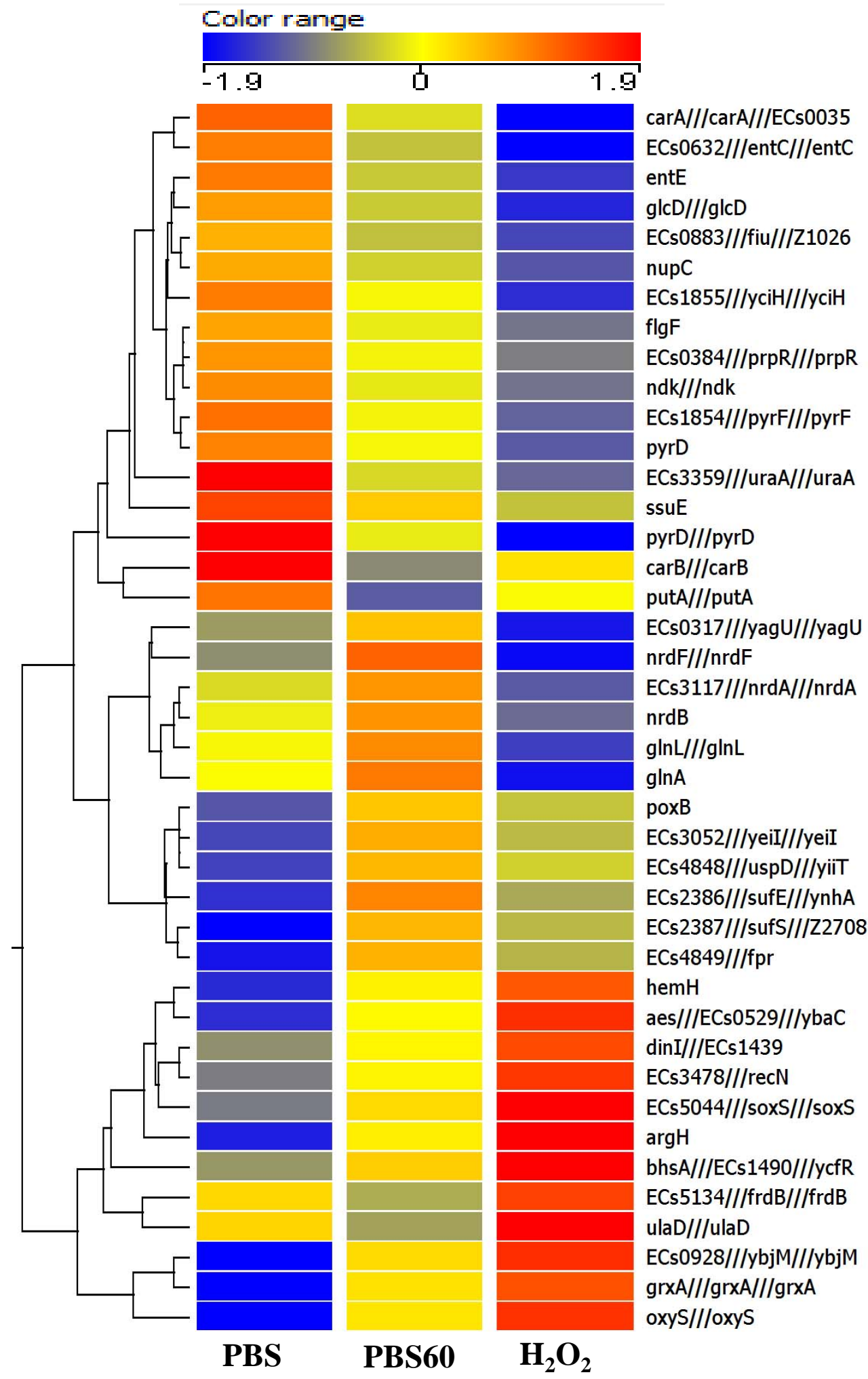

Figure 3. Hierarchical map of a comparison showing common genes that are differentially expressed in E. coli during exposure to plasma-activated PBS (PBS60) or $\mathrm{H}_{2} \mathrm{O}_{2}$ (oxidant; positive control), and compared with untreated PBS (PBS; negative control). Top 41 differentially expressed genes are shown here whose functional annotations are known. The functional annotation of most commonly influenced genes is shown in Table 1. 
Table 1. Functional analysis and category of differentially expressed genes involved in the response to plasma-activated PBS solution.

\begin{tabular}{|c|c|c|c|}
\hline Gene & Fold expression & Regulation & Function \\
\hline \multicolumn{4}{|c|}{ Oxidative damage response } \\
\hline oxyS & 28.8 & Up & Small regulatory RNA, responsive to oxidative stress \\
\hline katG & 19.3 & Up & Catalase/hydrogen peroxidase \\
\hline $\operatorname{sod} A$ & 2.1 & Up & Superoxide dismutase/detoxification of superoxide radicals \\
\hline $\operatorname{soxS}$ & 2.9 & Up & Transcriptional regulator of oxidative stress \\
\hline yhjA & 2.0 & Up & Cytochrome-C peroxidase activity \\
\hline$a h p F$ & 7.0 & Up & Disulfide oxidoreductase activity/peroxidase \\
\hline$a h p C$ & 3.9 & Up & Peroxidase activity/peroxiredoxin \\
\hline$y b i X$ & -2.4 & Down & Fe(II)-dependent oxygenase, hydroxylase activity \\
\hline \multicolumn{4}{|c|}{ SOS response } \\
\hline $\operatorname{recN}$ & 2.4 & Up & DNA recombination \& repair \\
\hline $\operatorname{din} L$ & 2.1 & Up & DNA-damage-inducible protein \\
\hline recD & 2.3 & Up & DNA repair, helicase activity, exonuclease V subunit \\
\hline $\operatorname{rec} B$ & 2.1 & Up & Exonuclease V subunit (recBCD complex) \\
\hline$n f o$ & 4.4 & Up & Endonuclease IV, DNA repair \\
\hline \multicolumn{4}{|c|}{ Nitrosative stress } \\
\hline hda & -3.2 & Down & DNA replication initiation factor \\
\hline napA & -2.6 & Down & Nitrate reductase, periplasmic \\
\hline napD & -3.2 & Down & Assembly protein for periplasmic nitrate reductase \\
\hline napF & -3.5 & Down & Ferredoxin-type protein, Fe-Fe binding, electron transport \\
\hline \multicolumn{4}{|c|}{ Related to cell envelop } \\
\hline ts $x$ & -2.5 & Down & Porin activity, iron membrane-transporter \\
\hline$y n b A$ & 2.2 & Up & Inner membrane, phosphotransferase \\
\hline yjcH & -2.2 & Down & Inner membrane protein, DUF485 family \\
\hline yagU & 2.7 & Up & Response to acidity, DUF1440 family \\
\hline yliE & 2.6 & Up & Hydrolase activity, inner membrane protein \\
\hline$f l g F$ & -2.0 & Down & Flagellar/motility, flagellum basal body \\
\hline$y c f S$ & -3.5 & Down & Peptidoglycan synthetase activity, cell shape \\
\hline $\operatorname{livJ}$ & -2.8 & Down & Carbon starvation induced gene, cell wall \\
\hline \multicolumn{4}{|c|}{ Other stress-related response } \\
\hline sufA & 2.4 & Up & Response to oxidative stress, Fe-S cluster protein \\
\hline $\operatorname{acn} A$ & 2.2 & Up & Aconitate hydratase/oxidative stress response/TCA cycle \\
\hline uspD & 5.1 & Up & Response to stress, cytoplamic protein \\
\hline cstA & -3.2 & Down & Response to stress, plasma membrane protein \\
\hline
\end{tabular}




\section{Continued}

\begin{tabular}{|c|c|c|c|}
\hline astD & -2.7 & Down & Succinylglutamic semialdehyde dehydrogenase, response to stress \\
\hline bhsA & -2.6 & Down & Biofilm, cell surface and signaling protein, response to stress \\
\hline$y m g C$ & 2.3 & Up & Biofilm formation, predicted protein \\
\hline$y h c N$ & -2.7 & Down & Cellular response to hydrogen peroxide, periplasmic space \\
\hline$y c f R$ & -2.6 & Down & Periplasmic protein, biofilm formation, response to stress \\
\hline \multicolumn{4}{|c|}{ Regulation/cell cycle/cell division } \\
\hline$g \ln L$ & 2.1 & Up & Histidine kinase activity, nitrogen regulation protein NR(II) \\
\hline$y c f S$ & -3.5 & Down & Transferase, peptidoglycan synthetase, transcriptional regulator \\
\hline malT & -3.3 & Down & Transcriptional regulator MalT \\
\hline napD & -3.2 & Down & Assembly protein for periplasmic nitrate reductase \\
\hline \multicolumn{4}{|c|}{ Metabolism } \\
\hline astA & -2.1 & Down & Acyltransferase, arginine N-succinyltransferase activity \\
\hline $\operatorname{prpR}$ & -2.1 & Down & Regulator for prp operon, propionate catabolism \\
\hline hcaR & -2.0 & Down & DNA-binding transcriptional regulator HcaR \\
\hline $\operatorname{Lip} A$ & 2.0 & Up & Lipoate synthase, transferase activity \\
\hline CarB & -14.5 & Down & Arginine biosynthetic process, synthase activity \\
\hline putA & -6.6 & Down & Proline dehydrogenase transcription \\
\hline pyrD & -6.2 & Down & Dihydroorotate dehydrogenase/UMP biosynthetic process \\
\hline $\operatorname{argH}$ & 4.6 & Up & Argininosuccinate lyase activity \\
\hline poxB & 4.1 & Up & Pyruvate oxidase, thiamine-dependent, FAD-binding \\
\hline hemH & 4.1 & Up & Porphyrin biosynthetic process, ferrochelatase \\
\hline purD & -3.5 & Down & Purine base biosynthetic process, synthase \\
\hline ycfS & -3.5 & Down & Peptidoglycan synthetase activity \\
\hline $\operatorname{car} A$ & -3.3 & Down & Glutamine amidotransferase, synthase activity \\
\hline entC & -3.3 & Down & Isochorismate synthase, enterobactin biosynthesis \\
\hline entE & -3.2 & Down & Enterobactin synthase, siderophore biosynthesis \\
\hline pyrB & -3.1 & Down & Aspartate carbamoyltransferase activity \\
\hline leuB & -3.1 & Down & Isopropylmalate dehydrogenase, amino acid synthesis \\
\hline Pyrl & -2.7 & Down & Transferase activity, pyrimidine biosynthesis \\
\hline$m d o D$ & -2.1 & Down & Osmoregulated glucan (OPG) biosynthesis, periplasmic protein \\
\hline alaC & 2.1 & Up & Valine-pyruvate aminotransferase activity \\
\hline$y g g F$ & -2.1 & Down & Fructose 1,6 bisphosphatase, glycerol metabolism \\
\hline aes & 3.9 & Up & Acetyl esterase, carboxylesterase activity \\
\hline$r f f H$ & -2.2 & Down & Extracellular polysaccharide biosynthetic process \\
\hline$r f f A$ & -2.2 & Down & Lipopolysaccharide biosynthetic process \\
\hline $\operatorname{arn} A$ & 2.0 & Up & Decarboxylase, lipid biosynthetic process \\
\hline
\end{tabular}




\section{Continued}

\begin{tabular}{|c|c|c|c|}
\hline$m d o D$ & -2.1 & Down & Osmoregulated glucan (OPG) biosynthesis \\
\hline$g a b D$ & -2.4 & Down & Succinate-semialdehyde dehydrogenase activity \\
\hline fumC & 2.4 & Up & Fumarate hydratase, TCA cycle \\
\hline$h t r A$ & -2.2 & Down & Serine endoprotease, proteolysis \\
\hline pncB & 2.2 & Up & Transferase activity, NAD biosynthetic process \\
\hline \multicolumn{4}{|c|}{ Fe-S cluster assembly/cysteine synthesis } \\
\hline$y f a E$ & 2.1 & Up & Ferredoxin metabolic process, 2Fe-2S ferredoxin \\
\hline ygfT & 2.6 & Up & Glutamate biosynthetic process, oxidoreductase, Fe-S subunit \\
\hline sufA & 2.4 & Up & Iron-sulfur cluster assembly scaffold protein \\
\hline$f r d B$ & -2.1 & Down & Fumarate reductase, Fe-S subunit \\
\hline hypC & -2.0 & Down & Hydrogenase assembly chaperone \\
\hline $\operatorname{grx} A$ & 10.8 & Up & Glutaredoxin 1, electron carrier activity \\
\hline sufD & 7.5 & Up & Cysteine desulfurase activator complex subunit SufD \\
\hline sufC & 5.5 & Up & Cysteine desulfurase ATPase component, ABC superfamily \\
\hline sufS & 8.4 & Up & Bifunctional cysteine desulfurase \\
\hline Fpr & 7.0 & Up & Ferredoxin-NADP reductase \\
\hline sufE & 3.5 & Up & Cysteine desufuration protein SufE, S-acceptor \\
\hline sufB & 3.7 & Up & Iron-sulfur cluster assembly , SufBCD complex \\
\hline fhuC & -2.1 & Down & Iron-hydroxamate transporter subunit \\
\hline $\operatorname{trx} C$ & 6.1 & $\mathrm{Up}$ & Thioredoxin-2 \\
\hline \multicolumn{4}{|c|}{ Transporters } \\
\hline$m n t H$ & 4.4 & Up & Manganese ion transmembrane transporter activity \\
\hline fepA & -2.1 & Down & Iron-enterobactin outer membrane transporter \\
\hline$f \operatorname{tn} B$ & -2.2 & Down & Ferritin-like protein, cellular iron ion homeostasis \\
\hline $\operatorname{lsr} C$ & 2.1 & Up & Transport system permease protein \\
\hline $\arg H$ & 4.6 & Up & Cellular amino acid biosynthetic process \\
\hline$g \ln A$ & 2.3 & Up & Glutamine synthetase \& transport regulation, N2-deprivation \\
\hline putP & -3.7 & Down & Major sodium/proline symporter \\
\hline cmtA & 2.6 & Up & Carbohydrate transport, sugar:hydrogen symporter \\
\hline$m n g A$ & 2.4 & Up & Carbohydrate transporter, IIABC components system \\
\hline $\operatorname{malK}$ & 2.0 & Up & Maltose/maltodextrin transporter \\
\hline$m d l B$ & 2.3 & Up & Multidrug transporter \\
\hline$n m p C$ & -4.8 & Down & Outer membrane porin protein, nmpC \\
\hline$d p p C$ & -2.4 & Down & Dipeptide/heme transporter \\
\hline narU & 5.1 & $\mathrm{Up}$ & Nitrate/nitrite transporter protein \\
\hline $\operatorname{araE}$ & 2.3 & Up & Arabinose-proton symporter, carbohydrate transporter \\
\hline $\operatorname{dct} A$ & -3.4 & Down & Sodium:dicarboxylate symporter activity \\
\hline
\end{tabular}


was less pronounced. Other stress-responsive universal genes which were differentially expressed include acnA (TCA cycle), uspD (cytoplasmic protein), cstA (plasma membrane protein), and yhcN (periplasmic space protein). The gene bhsA (formerly ycfR) that mediate biofilm formation was downregulated. Similarly, most of the genes encoding the proteins from biosynthesis and metabolic pathways were significantly downregulated (Table 1), and indicated that cells under (ROS or RNS) stress-induced adverse condition, indicating a general energy depletion, and conversely minimized energy consumption in order to prioritize survival. The expression of transcripts of ycfS (peptidoglycan synthetase), malT (MalT system), and napD (nitrate reductase) were significantly downregulated.

\subsection{Cell Envelop and Membrane Associated and Transport Associated Genes}

The transporter of manganese $(m n t H)$ was upregulated $>4$ folds, while almost all iron mediating transporters and related protein transcription, such as fepA (iron-enterobactin) ftnB (ferritin-like protein involved in cellular iron ion homeostasis), fepC (iron-enterobactin, ATP-binding subunit), $d p p C$ (dipeptide, heme transporter), napF (ferredoxin type protein) were downregulated. This suggests a disrupted iron homeostasis. The transporters of glucarate, glutamine, sugar:hydrogen, carbohydrate, maltodextrin, multidrug, amino acid, nitrate-nitrite were all upregulated (Table 1). The genes of outer membrane porin, nmpC, and iron membrane porin activity protein, $t s x$ were downregulated.

\section{Discussion}

Despite of increasing reports on application of nonthermal plasma in disinfection and bacterial inactivation, relatively very little is known about the stress response of bacterial cell to it. In this study, we report gene expression profile and transcriptomic responses of E. coli to plasma-activated PBS solution which inactivates bacterial cell [7]. Three minutes plasma-treated PBS solution completely inactivated E. coli upon contact time of 15 minutes [7], and therefore not shown here. On the basis of our earlier observations, and the specific protection provided by ROS scavengers (Figure 1), we anticipated that this solution would mount oxidative stress in bacterial cell, and might be containing a mixture of ROS, including a stabilized species such as hydrogen peroxide [7] [8]. The amount of $\mathrm{H}_{2} \mathrm{O}_{2}$ generated in treated PBS at 60 seconds of plasma-activated of PBS was much smaller (0.42 $\mathrm{mM}$ ) as compared to originally estimated $0.01 \%$ of $\mathrm{H}_{2} \mathrm{O}_{2}$ standard solution which corresponded to $3.26 \mathrm{mM}$. Hydrogen peroxide is a known antimicrobial agent. However, a concentration of $\mathrm{H}_{2} \mathrm{O}_{2}$ required to complete inactivation of most bacteria is as low as $124 \mathrm{mM}(0.38 \%)$. Therefore, results suggest indirectly that other reactive species in the solution were present which participate in the inactivation process (either as added or synergistic effect) [7] [8]. Hence it is likely that oxidative stress mediating transcriptional response by plasma-activated PBS though not completely, would be little different as compared to $\mathrm{H}_{2} \mathrm{O}_{2}$ in type and intensity.

An analysis using global gene expression approach is recently used by Dr. Pruden's Laboratory [6] using argon gas plasma (an entirely different type of plasma set up, and parameters), where an overlapping expression patterns were observed; therefore this study becomes more interesting. Our microarray data analysis suggests that selection of hydrogen peroxide as a positive control for this study was logical. Hydrogen peroxide is known to generate reactive oxygen species and activate related pathways in E. coli [14].

\subsection{SOS Response and Distress Signals}

The SOS responses in E. coli are induced after DNA damage, and are dynamically regulated by interplay between Rec family protein, such as RecA, Lex protein, LexA, and Sul protein, SulA. RecN is a conserved SMClike (structural maintenance of chromosomes) nucleoid-associated ATPase involved in the tethering of chromatids and in double strand break (DSB) repairs [15], and thus involved in DNA recombination and repair. RecN plays a crucial role in homologous recombination-dependent DSB repair, and it is required upstream of RecA-mediated strand exchange. RecN is also highly conserved in E. coli and it has two SOS boxes in its promoter region. Therefore, the expression of $r e c N$ is tightly regulated by LexA repressor [16]. In E. coli, DSB repair is initiated by RecBCD, a main double-strand exonuclease. The transcription of recN, recB and recD components was increased in the present study. Operon $n r d A B$ and $n r d E F$ encoding ribonucleotide reductases which supply deoxynucleoside triphosphate (dNTP) substrates for DNA replication were found upregulated (Table 1). Endonuclease IV encoded by $n f o$ is also involved in DNA repair, and is a component of SoxRS regulon system, and 
activated by SoxS superoxide-responsive regulatory protein. Thus SOS response and oxidative stress are reportedly related [17].

\subsection{Oxidative Stress Response Is Predominant}

E. coli has several major regulators that are activated during oxidative stress and undergo conformational changes, but two regulon systems are important in oxidative stress management. These are OxyR and SoxR transcriptional regulators, sensitive to oxidation in presence of hydrogen peroxide $\left(\mathrm{H}_{2} \mathrm{O}_{2}\right)$ and superoxide radicals $\left(\mathrm{O}_{2}^{-}\right)$respectively and subsequently regulate the expression of an array of cognate genes. ROS are the inevitable by-product of oxidative stress and oxidation, and the species such as $\mathrm{H}_{2} \mathrm{O}_{2}$ and $\mathrm{O}_{2}^{-}$are generated when they react with flavin cofactors containing dehydrogenases and other cell components. Reactive nitric oxide is also generated as denitrification intermediate, and can further react with superoxide or hydroxyl $(\cdot \mathrm{OH})$ radicals. Reactive $\cdot \mathrm{OH}$ often results from $\mathrm{H}_{2} \mathrm{O}_{2}$ interaction with unincorporated ferrous iron by Fenton chemistry [18]. OxyS is a small regulatory RNA, responsive to oxidative stress, especially $\mathrm{H}_{2} \mathrm{O}_{2}$ rich environment where it detoxifies the latter. We observed highest expression of oxyS transcript (Table 1), an indicator of a generation of severe oxidative stress, and may suggest a synergy of different reactive oxidative species that enhance inactivation of $E$. coli cells during plasma-activated PBS exposure. Similar observation was noted by Pruden Laboratory [6] during direct argon gas plasma application, indicating that oxidative stress is observed in both, direct plasma exposure and plasma-activated fluid. OxyS further regulates multiple targets (over a dozen genes); of which so$d A$ is one of the major players that was upregulated in our study. Sox regulon is also closely related with Mar regulon genes, but we did not see any differential regulation of the latter in our study. SoxR has been shown to detect superoxide via oxidation of its iron-sulfur cluster, and OxyR detects $\mathrm{H}_{2} \mathrm{O}_{2}$ via oxidation of specific cysteine residues. The important members of OxyR-regulated genes, such as ahpC, $a h p F, k a t G, \operatorname{grx} A, \operatorname{trx} C$ and gor were upregulated both in the conditions of plasma-treated PBS solution and hydrogen peroxide control. Together these findings suggest the generation of reactive oxygen species, such as hydrogen peroxide and superoxide.

\subsection{Other Stress-Related Genes and Their Metabolic Responses}

Cells under (ROS or RNS) stress often activate transcription factor OxyR which in turn regulates the expression of a large panel of genes, including a small regulatory RNA, oxyS, as mentioned above. Such observations are reported during severe oxidative as well as nitrosative stress conditions [19] [20], and in argon gas plasma system [3] [6]. We observed reactive nitrogen species-responsive genes [13] such as hda and napA were downregulated, and the genes involved in Fe-S cluster assembly and sulfur acquisition were significantly upregulated. The hda encode for DNA replication initiation factor, and is the first $E$. coli chromosomal gene shown to initiate translation with CTG. Nap enzyme complex is localized to the periplasm with NapA, NapB and NapC, and identified as the essential components for nitrate reduction in $E$. coli. NapA is enzymatically active subunit that contains Fe-S cluster and molybdenum co-factor; reduces $\mathrm{NO}_{3}$ to $\mathrm{NO}_{2}$. RNS function as powerful antimicrobials in both the mammalian host cell and bacterial cells [13]. Bacteria developed certain mechanisms to survive under nitrosative stress by the mechanisms such as RNS-mediated protein modifications, reversible binding of nitric oxide to multiple Fe-S clusters or thiol groups [21]. More than 110 proteins in $E$. coli require Fe/S clusters to function, where they control enzyme catalysis, electron transport, and regulation of gene expression. During in-vitro, large amounts of free iron and sulfide become available spontaneously in assembly of Fe/S clusters, however in-vivo these elements are toxic to cells, and hence concentrations are tightly regulated [22]. Bacteria utilize at least one of the three systems known to assemble such clusters, namely Isc, Suf and Nif systems, of which former two (mediated by isc and suf operons) are reportedly induced under ROS/RNS mediated oxidative stress conditions. The suf (sufABCDSE) operon encoding a Fe-S assembly system is induced by peroxides through activators OxyR and IscR in E. coli [23]-[25], and is an alternative system (to Isc) under oxidative and Fe-limiting conditions. In the present study only Suf system was found differentially expressed, and indicates that plasma-activated PBS probably produce peroxides in sufficient amount, required for activation of suf operon. Our earlier findings on exposure of $E$. coli cells to direct plasma discharge (not through plasma-treated fluid) also demonstrated the generation of hydrogen peroxide, and the specific scavenger such as catalase was able to decompose peroxide significantly [3]. The present microarray analysis is therefore important in this context, and further studies are required for comparing the exact differences. 


\subsection{Plasma-Treated PBS Solution Influence Cell Envelop and Membrane Associated Mechanisms}

It can be predicted that the interaction of ROS generated by plasma-treated solution exposure to cellular iron may have potential detrimental effect on bacteria, either by unavailability of iron for bacterial cell (iron starvation) or leading to series of lethal reactions that further regenerate hydroxyl radical (such as Fenton reaction) [26]-[28], influencing the expression of several important functional genes. The genes encoding most carbohydrate/sugar transporters were upregulated, whereas the ones for biosynthesis were downregulated. Plasmaactivated PBS solution had downregulated genes involved in flagella, motility, fimbriae, adhesin, and quorum sensing (Table 1). Bacterial biofilm formation and cell motility are intrinsically correlated [29], and both compromised by oxidative stress, acid stress or starvation [30], and differentially expressed, depending upon the amount of stress. A transpeptidase, the protein (encoded by $y c f S$ ) required for peptidoglycan synthesis was down-regulated during plasma-treated PBS, and indicates that the energy involving processes are minimized by E. coli under such stress. In addition to ROS/RNS generated in plasma-treated PBS, the solution became acidic [7]. We demonstrated earlier that antimicrobial property is not due to a mere change in $\mathrm{pH}$ but a likely synergy effect of all reactive species and concurrent reduction in $\mathrm{pH}$. Acid-induced genes are associated with increased catabolic processes, generate oxidative stress, activate heat shock regulons, and differentially regulate large number of proton gradient, periplasmic and cell envelope proteins, energy-driven synthesis and assembly of flagella.

Under oxidative stress in E. coli several transporters are differentially expressed such as iron transporters. Iron is required for many metabolic processes and plays a role in protection against oxidative damage. However, excess iron levels in cells contribute to oxidative damage through the generation of free radicals [18]. Global regulators such as OxyR and SoxR have an association with their co-repressor that represses transcription of genes encoding high-affinity transport systems and other related proteins involved in iron metabolism, and thus tightly control iron hemeostasis. We observed a downregulation of genes that encodes iron transporting and binding proteins, such as tsx (iron membrane transporter exhibit porin like activity), fepA (iron-enterobactin outer membrane transporter), fhuC (iron-hydroxamate transporter subunit), $f \operatorname{tn} B$ (ferritin-like protein having iron ion homeostasis) and fepC (the ATP-binding subunit of iron-enterobactin transporter (Table 1). In contrary, manganese ion transporter encoded by $\mathrm{mntH}$, and manganese-dependent superoxide dismutase encoded by sodA were found upregulated. Thus control of both, the iron and manganese transport, and Fe- and Mn-dependent defense against oxidative stress could be well coordinated [31]. Further details of the mechanisms are required to be investigated.

\section{Conclusion}

This transcriptomic study suggests that E. coli cells differentially express several important genes that are responsive to oxidative stress generated upon exposure to plasma-activated PBS solution. The genes responsive to hydrogen peroxide, superoxide and singlet oxygen, and reactive nitrogen species were observed, and might be collectively exerting their damaging effect on E. coli cell. The data presented here are predictive and for the guidance, and further detailed studies would be interesting to elucidate the exact nature of responses to different nonthermal plasma set-ups and their various parameters.

\section{Acknowledgements}

Adam Yost (graduate) and Siddharth Joshi (undergraduate) research students at School of Biomedical Engineering, Drexel University, and Suresh G. Joshi, Garth Ehrlich and Ari Brooks, the faculties at Center for Surgical Infection and Biofilm, Drexel University College of Medicine, and Sankar Addya, the in-charge of Microarray Core Facility at Thomas Jefferson University, Philadelphia, PA. All authors contributed to this manuscript, and approved the final version of it. SGJ designed the study; SGJ, AY, SSJ, and SA carried out the experiments; SGJ and SA analyzed preliminary data; SGJ, SA, GE and AB interpreted data from multiple ways; SGJ developed the manuscript draft; and SGJ, AB and GE evaluated the manuscript. This study was supported by the Department of Surgery through Surgical Infection Research Program. The authors thank Microarray Core Facility of Kimmel Cancer Center, Philadelphia, PA. 


\section{Competing Interest}

The authors declare "no conflict" and no competing interests.

\section{References}

[1] Burts, M.L., Alexeff, I., Meek, E.T. and McCullers, J.A. (2009) Use of Atmospheric Non-Thermal Plasma as a Disinfectant for Objects Contaminated with Methicillin-Resistant Staphylococcus aureus. American Journal of Infection Control, 37, 729-733. http://dx.doi.org/10.1016/j.ajic.2009.03.010

[2] Kvam, E., Davis, B., Mondello, F. and Garner, A.L. (2012) Nonthermal Atmospheric Plasma Rapidly Disinfects Multidrug-Resistant Microbes by Inducing Cell Surface Damage. Antimicrobial Agents and Chemotherapy, 56, $2028-2036$. http://dx.doi.org/10.1128/AAC.05642-11

[3] Joshi, S.G., Cooper, M., Yost, A., Paff, M., Ercan, U.K., Fridman, G., Friedman, G., Fridman, A. and Brooks, A.D. (2011) Nonthermal Dielectric-Barrier Discharge Plasma-Induced Inactivation Involves Oxidative DNA Damage and Membrane Lipid Peroxidation in Escherichia coli. Antimicrobial Agents and Chemotherapy, 55, 1053-1062. http://dx.doi.org/10.1128/AAC.01002-10

[4] Cooper, M., Fridman, G., Fridman, A. and Joshi, S.G. (2010) Biological Responses of Bacillus stratosphericus to Floating Electrode-Dielectric Barrier Discharge Plasma Treatment. Journal of Applied Microbiology, 109, 2039-2048. http://dx.doi.org/10.1111/j.1365-2672.2010.04834.x

[5] Desmet, T., Morent, R., De Geyter, N., Leys, C., Schacht, E. and Dubruel, P. (2009) Nonthermal Plasma Technology as a Versatile Strategy for Polymeric Biomaterials Surface Modification: A Review. Biomacromolecules, 10, 23512378. http://dx.doi.org/10.1021/bm900186s

[6] Sharma, A., Collins, G. and Pruden, A. (2009) Differential Gene Expression in Escherichia coli Following Exposure to Nonthermal Atmospheric Pressure Plasma. Journal of Applied Microbiology, 107, 1440-1449. http://dx.doi.org/10.1111/j.1365-2672.2009.04323.x

[7] Ercan, U.K., Wang, H., Ji, H.F., Fridman, G., Brooks, A.D. and Joshi, S.G. (2013) Nonequilibrium Plasma-Activated Antimicrobial Solutions Are Broad-Spectrum and Retain Their Efficacies for Extended Period of Time. Plasma Processes and Polymers, 10, 544-555. http://dx.doi.org/10.1002/ppap.201200104

[8] Kojtari, A., Ercan, U.K., Smith, J., Friedman, G., Sensenig, R.B., Tyagi, S., Joshi, S.G., Ji, H.-F. and Brooks, A.D. (2013) Chemistry for Antimicrobial Properties of Water Treated with Non-Equilibrium Plasma. Nanomedicine and Biotherpaeutic Discovery, 4, 1-5.

[9] Imlay, J.A. (2013) The Molecular Mechanisms and Physiological Consequences of Oxidative Stress: Lessons from a Model Bacterium. Nature Reviews Microbiology, 11, 443-454. http://dx.doi.org/10.1038/nrmicro3032

[10] Eisen, M.B. and Brown, P.O. (1999) DNA Arrays for Analysis of Gene Expression. Methods in Enzymology, 303, 179205. http://dx.doi.org/10.1016/S0076-6879(99)03014-1

[11] Joshi, S.G., Paff, M., Friedman, G., Fridman, G., Fridman, A. and Brooks, A.D. (2010) Control of Methicillin-Resistant Staphylococcus aureus in Planktonic Form and Biofilms: A Biocidal Efficacy Study of Nonthermal Dielectric-Barrier Discharge Plasma. American Journal of Infection Control, 38, 293-301.

http://dx.doi.org/10.1016/j.ajic.2009.11.002

[12] Perumal Samy, R., Pachiappan, A., Gopalakrishnakone, P., Thwin, M.M., Hian, Y.E., Chow, V.T., Bow, H. and Weng, J.T. (2006) In Vitro Antimicrobial Activity of Natural Toxins and Animal Venoms Tested against Burkholderia pseudomallei. BMC Infectious Diseases, 6, 100. http://dx.doi.org/10.1186/1471-2334-6-100

[13] Vercaigne, L.M., Sitar, D.S., Penner, S.B., Bernstein, K., Wang, G.Q. and Burczynski, F.J. (2000) Antibiotic-Heparin Lock: In Vitro Antibiotic Stability Combined with Heparin in a Central Venous Catheter. Pharmacotherapy, 20, 394399. http://dx.doi.org/10.1592/phco.20.5.394.35063

[14] Sobota, J.M., Gu, M. and Imlay, J.A. (2014) Intracellular Hydrogen Peroxide and Superoxide Poison 3-Deoxy-D-Arabinoheptulosonate 7-Phosphate Synthase, the First Committed Enzyme in the Aromatic Biosynthetic Pathway of Escherichia coli. Journal of Bacteriology, 196, 1980-1991. http://dx.doi.org/10.1128/JB.01573-14

[15] Booth, J.A., Thomassen, G.O., Rowe, A.D., Weel-Sneve, R., Lagesen, K., Kristiansen, K.I., Bjoras, M., Rognes, T. and Lindvall, J.M. (2013) Tiling Array Study of MNNG Treated Escherichia coli Reveals a Widespread Transcriptional Response. Scientific Reports, 3, 3053. http://dx.doi.org/10.1038/srep03053

[16] Rostas, K., Morton, S.J., Picksley, S.M. and Lloyd, R.G. (1987) Nucleotide Sequence and LexA Regulation of the Escherichia coli recN Gene. Nucleic Acids Research, 15, 5041-5049. http://dx.doi.org/10.1093/nar/15.13.5041

[17] Li, Z. and Demple, B. (1994) SoxS, an Activator of Superoxide Stress Genes in Escherichia coli. Purification and Interaction with DNA. The Journal of Biological Chemistry, 269, 18371-18377.

[18] Imlay, J.A. (2008) Cellular Defenses against Superoxide and Hydrogen Peroxide. Annual Review of Biochemistry, 77, 
755-776. http://dx.doi.org/10.1146/annurev.biochem.77.061606.161055

[19] Jozefczuk, S., Klie, S., Catchpole, G., Szymanski, J., Cuadros-Inostroza, A., Steinhauser, D., Selbig, J. and Willmitzer, L. (2010) Metabolomic and Transcriptomic Stress Response of Escherichia coli. Molecular Systems Biology, 6, 364. http://dx.doi.org/10.1038/msb.2010.18

[20] Zuber, P. (2009) Management of Oxidative Stress in Bacillus. Annual Review of Microbiology, 63, 575-597. http://dx.doi.org/10.1146/annurev.micro.091208.073241

[21] Boyd, J.M., Lewis, J.A., Escalante-Semerena, J.C. and Downs, D.M. (2008) Salmonella enterica Requires ApbC Function for Growth on Tricarballylate: Evidence of Functional Redundancy between ApbC and IscU. Journal of Bacteriology, 190, 4596-4602. http://dx.doi.org/10.1128/JB.00262-08

[22] Tsaousis, A.D., Ollagnier de Choudens, S., Gentekaki, E., Long, S., Gaston, D., Stechmann, A., Vinella, D., Py, B., Fontecave, M., Barras, F., Lukes, J. and Roger, A.J. (2012) Evolution of Fe/S Cluster Biogenesis in the Anaerobic Parasite Blastocystis. Proceedings of the National Academy of Sciences of the United States of America, 109, 1042610431. http://dx.doi.org/10.1073/pnas.1116067109

[23] Lee, K.C., Yeo, W.S. and Roe, J.H. (2008) Oxidant-Responsive Induction of the Suf Operon, Encoding a Fe-S Assembly System, through Fur and IscR in Escherichia coli. Journal of Bacteriology, 190, 8244-8247. http://dx.doi.org/10.1128/JB.01161-08

[24] Kullik, I., Stevens, J., Toledano, M.B. and Storz, G. (1995) Mutational Analysis of the Redox-Sensitive Transcriptional Regulator OxyR: Regions Important for DNA Binding and Multimerization. Journal of Bacteriology, 177, $1285-1291$.

[25] Kullik, I., Toledano, M.B., Tartaglia, L.A. and Storz, G. (1995) Mutational Analysis of the Redox-Sensitive Transcriptional Regulator OxyR: Regions Important for Oxidation and Transcriptional Activation. Journal of Bacteriology, 177, 1275-1284.

[26] Luo, Y., Henle, E.S., Gassmann, W. and Linn, S. (1996) Oxidative Damage to DNA Constituents by Iron-Mediated Fenton Reactions. The Deoxyguanosine Family. The Journal of Biological Chemistry, 271, 21177-21186. http://dx.doi.org/10.1074/jbc.271.35.21177

[27] Andrews, S.C., Robinson, A.K. and Rodriguez-Quinones, F. (2003) Bacterial Iron Homeostasis. FEMS Microbiology Reviews, 27, 215-237. http://dx.doi.org/10.1016/S0168-6445(03)00055-X

[28] Liu, I.F., Annamalai, T., Sutherland, J.H. and Tse-Dinh, Y.C. (2009) Hydroxyl Radicals Are Involved in Cell Killing by the Bacterial Topoisomerase I Cleavage Complex. Journal of Bacteriology, 191, 5315-5319. http://dx.doi.org/10.1128/JB.00559-09

[29] Houry, A., Briandet, R., Aymerich, S. and Gohar, M. (2010) Involvement of Motility and Flagella in Bacillus cereus Biofilm Formation. Microbiology, 156, 1009-1018. http://dx.doi.org/10.1099/mic.0.034827-0

[30] Lemke, J.J., Durfee, T. and Gourse, R.L. (2009) DksA and ppGpp Directly Regulate Transcription of the Escherichia coli Flagellar Cascade. Molecular Microbiology, 74, 1368-1379. http://dx.doi.org/10.1111/j.1365-2958.2009.06939.x

[31] Sabri, M., Leveille, S. and Dozois, C.M. (2006) A SitABCD Homologue from an Avian Pathogenic Escherichia coli Strain Mediates Transport of Iron and Manganese and Resistance to Hydrogen Peroxide. Microbiology, 152, 745-758. http://dx.doi.org/10.1099/mic.0.28682-0 
Scientific Research Publishing (SCIRP) is one of the largest Open Access journal publishers. It is currently publishing more than 200 open access, online, peer-reviewed journals covering a wide range of academic disciplines. SCIRP serves the worldwide academic communities and contributes to the progress and application of science with its publication.

Other selected journals from SCIRP are listed as below. Submit your manuscript to us via either submit@scirp.org or Online Submission Portal.
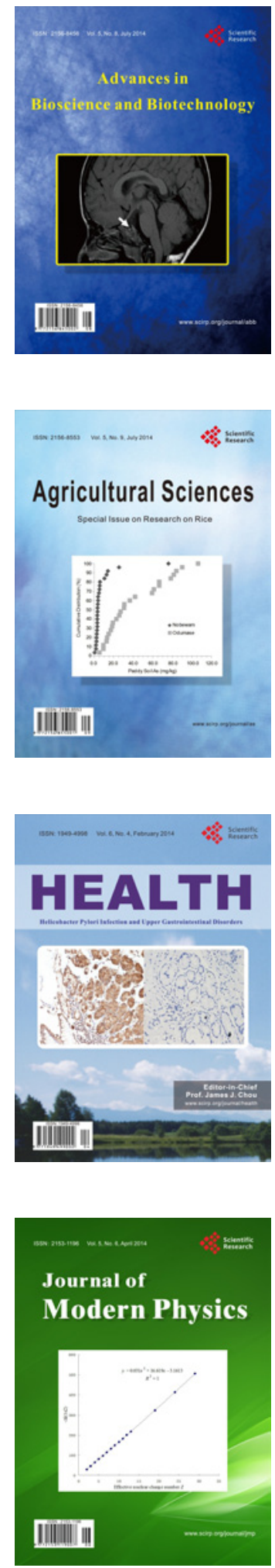
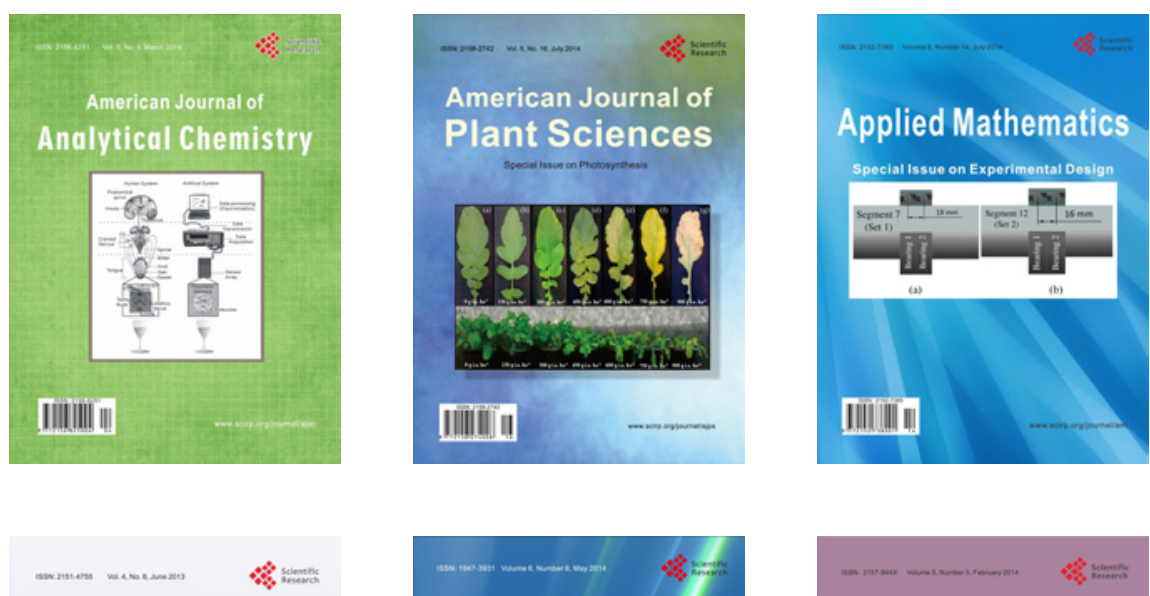

Creative Education
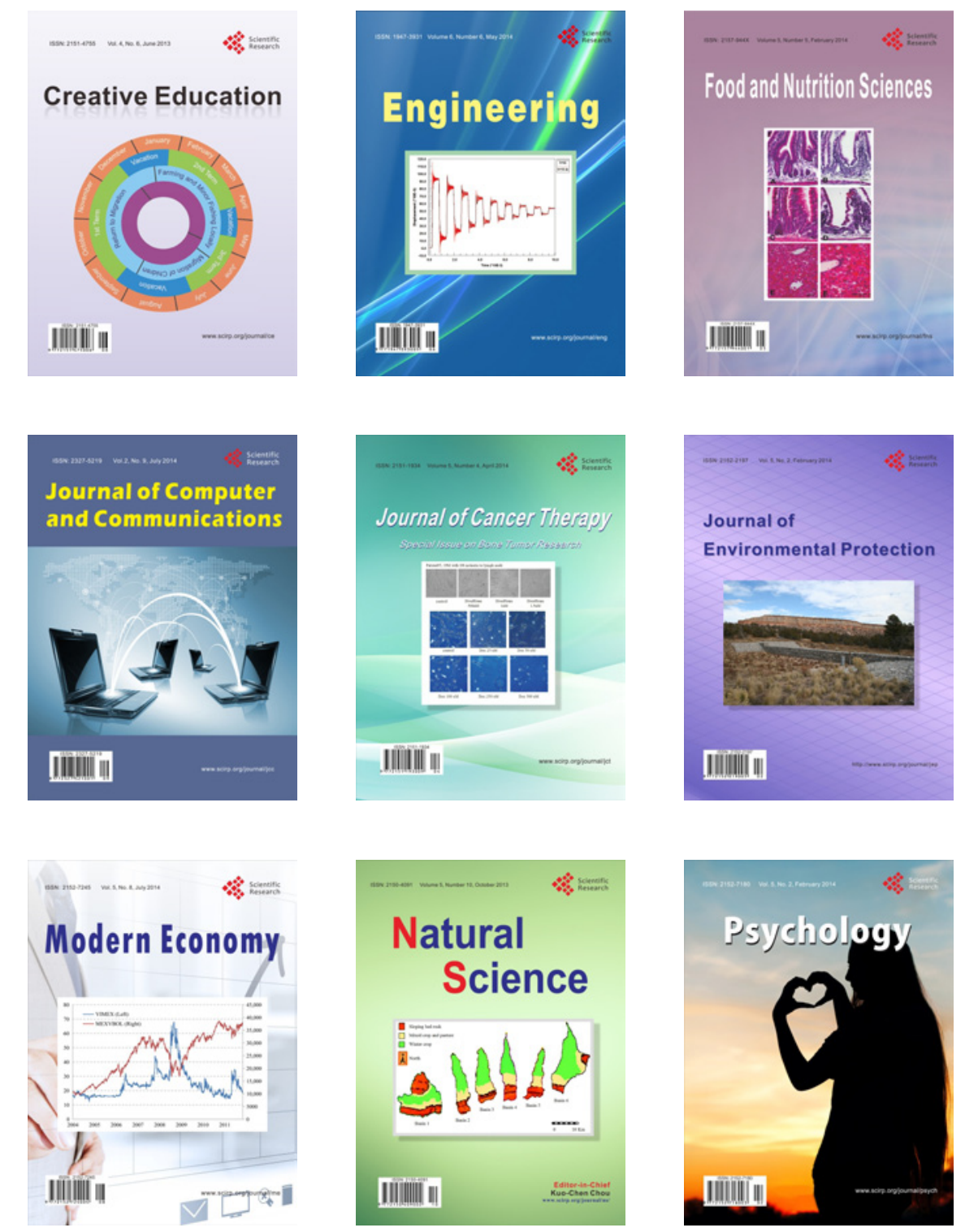